\title{
LETTER
}

\section{Long term outcomes of critically ill COVID-19 pneumonia patients: early learning}

\author{
Claire McCue ${ }^{1}$, Richard Cowan ${ }^{1}$, Tara Quasim ${ }^{1,2}$, Kathryn Puxty $^{1,2}$ and Joanne McPeake ${ }^{1,2^{*}}$ (D)
}

(0) 2020 Springer-Verlag GmbH Germany, part of Springer Nature

\section{Dear Editor,}

Patients treated for coronavirus disease 2019 (COVID19) pneumonia in the intensive care unit (ICU) often experience long periods of ventilation, neuromuscular blockade and sedation [1]. Previous research has demonstrated that patients with similar clinical journeys often have poor long-term health related quality of life (HRQoL) [2]. At present there are limited data describing the long-term outcomes of critically ill COVID-19 survivors. To address this, we report on early data obtained at our ICU follow-up programme in a single centre.

Patients are routinely invited to our multi-disciplinary ICU follow-up clinic between 12-16 weeks post discharge [3]. Information on the format of the clinic is available in S1. Data were collected following attendance at a virtual clinic. HRQoL was measured using the EQ5D-5L. This tool comprises two sections: a five-question descriptive component which explores health domains and a visual analogue scale about HRQoL. Each question has five possible answers. These answers produce a fivedigit sequence which is used to determine a health utility score (HUS). A HUS of 1 equates to the best health state possible, 0 with death and a negative HUS equates to a state worse than death [4]. We also examined return to employment. Ethical approval was granted by The North West (Liverpool Central) Research Ethics Committee, REC Number: 17/NM/0199. All patients provided consent.

From March 14th, 2020 until April 28th, 2020, 51 patients required invasive mechanical ventilation for COVID-19 pneumonia in our ICU. All cases were

\footnotetext{
*Correspondence: joanne.mcpeake@glasgow.ac.uk

${ }^{2}$ School of Medicine, Dentistry and Nursing, University of Glasgow, Glasgow, UK
}

Full author information is available at the end of the article confirmed with reverse RT-PCR assay for SARS-CoV-2, except for one patient who died prior to a sputum sample being obtained. Complete records of 43 patients were available for review. Of the 43 patients examined, 33 (77\%) were male and the median age was 57 (IQR 52.565.5). 27 (63\%) patients had 1 or more comorbidity. ICU mortality was $33 \%$, median ICU length of stay 17.9 days (IQR 7.4-26.5) and the median duration of ventilation was also 17.9 (IQR 6.5-24). Most (93\%) patients developed severe acute respiratory distress syndrome (ARDS) during their admission $(\mathrm{P} / \mathrm{F}$ or $\mathrm{S} / \mathrm{F}$ ratio $<13.3 \mathrm{kPa}$ ) and $37(86 \%)$ received neuromuscular blockade with a median of 6 (IQR 2.5-11) days paralysis. Sixty percent of patients were proned at least once (Table 1 ).

Of the 30 survivors with full data available, $24(80 \%)$ attended follow up. Outcome data were available from 21 patients; one patient declined inclusion; one was readmitted to hospital following their consultation and could not participate in the research and one agreed to participate but could not be contacted following clinic. The median HUS was 0.752 (IQR 0.627-0.837). Fifteen patients were employed pre-ICU. When reviewed at follow-up, 7 (47\%) had returned to work and $1(7 \%)$ had taken voluntary retirement. One $(7 \%)$ patient was planning to return to work in the week following clinic attendance. New disability including breathlessness were reported as impacting on employability; a small number of patients described they were unable to return to work due to COVID-19 employment restrictions. Fourteen (67\%) patients complained of new pain; $29 \%$ of pain was classified as severe or extreme.

This cohort had a significant burden of acute illness requiring prolonged mechanical ventilation and high rates of neuromuscular blockade. Despite this, we report meaningful early recovery including increased return to employment in a single centre, from a small sample [5]. However, caution should be taken with the interpretation

\section{Springer}


Table 1 ICU cohort demographics

\begin{tabular}{ll}
\hline Demographic & $n=43$ \\
\hline Age, year, median (IQR) & $57(52.5-65.5)$ \\
Gender, male (\%) & $33(77 \%)$ \\
Comorbidity: & \\
\hline Diabetes & $9(21 \%)$ \\
Respiratory & $8(19 \%)$ \\
Cardiovascular (including hypertension) & $13(30 \%)$ \\
\hline Other (cancer, liver disease, CKD) & $12(28 \%)$ \\
Length of ICU stay, days, median (IQR) & $17.9(7.4-26.5)$ \\
Mechanical ventilation duration, days, median (IQR) & $17.9(6.5-24)$ \\
Diagnosis of severe ARDS (\%) & $40(93 \%)$ \\
Neuromuscular blockage administration (\%) & $37(86 \%)$ \\
Prone position (\%) & $26(60 \%)$ \\
\hline
\end{tabular}

of these outcomes, as employment status can fluctuate following critical illness [5]. Furthermore, HRQoL was similar to a previous ARDS cohort (Median HUS, 0.77) and better than a previous cohort of survivors from our own centre (Median HUS, 0.29) [3, 6]. Two thirds of patients experienced ongoing, new pain following discharge, a finding which requires further investigation.

\section{Electronic supplementary material}

The online version of this article (https://doi.org/10.1007/s00134-020-06313-x) contains supplementary material, which is available to authorized users.

\section{Author details}

${ }^{1}$ Intensive Care Unit, Glasgow Royal Infirmary, Glasgow, UK. ${ }^{2}$ School of Medicine, Dentistry and Nursing, University of Glasgow, Glasgow, UK.

\section{Funding}

JM is funded by a THIS. Institute, University of Cambridge Research Fellowship (PD-2019-02-16).

\section{Compliance with ethical standards}

\section{Conflicts of interest}

The authors have no conflicts of interest to declare.

\section{Publisher's Note}

Springer Nature remains neutral with regard to jurisdictional claims in published maps and institutional affiliations.

\section{Accepted: 23 October 2020}

Published online: 9 November 2020

\section{References}

1. Ferrando C, Suarez-Sipmann F, Mellado-Artigas R et al (2020) Clinical features, ventilatory management, and outcome of ARDS caused by COVID-19 are similar to other causes of ARDS. Intensive Care Med. https ://doi.org/10.1007/s00134-020-06192-2

2. Herridge MSM, Hough M et al (2016) Recovery and outcomes after acute respiratory distress syndrome (ARDS) in patients and their family caregivers. Intensive Care Med 42:725-738

3. McPeake JM, Shaw M, Iwashyna TJ et al (2017) Intensive care syndrome: promoting independence and return to employment (InS:PIRE). Early evaluation of a complex intervention. PLoS ONE 12(11):e0188028

4. Sullivan T, Hansen P, Ombler F et al (2020) A new tool for creating personal and social EQ-5D-5L value sets, including value 'dead.'Soc Sci Med 246:112707

5. McPeake JM, Mikkelsen ME, Quasim T, et al (2019) Return to employment following critical illness and its association with psychosocial outcomes: a systematic review and meta-analysis. Ann Am ThoracSoc 16(10):1304-1311

6. Brown SM, Wilson E, Presson AP et al (2017) Predictors of 6-month health utility outcomes in survivors of acute respiratory distress syndrome. Thorax 72:311-317 